\title{
Novel Zeolite Modified Carbon Paste Electrode for Differential Pulse Voltammetric Assay of Cyclopentolate hydrochloride
}

\author{
Salhah D. Al-Qahtani ${ }^{1}$, Ahmed Hameed ${ }^{2}$,Enas Aljuhani ${ }^{2}$, Ali Sayqal ${ }^{2}$, Reem Shah ${ }^{2}$, Fawaz Saad ${ }^{2}$, \\ Nashwa M. El-Metwaly ${ }^{2,3 *}$ \\ ${ }^{1}$ Department of Chemistry, College of Science, Princess Nourah bint Abdulrahman University, \\ Riyadh, Saudi Arabia \\ ${ }^{2}$ Department of Chemistry, Faculty of Applied Science, Umm-Al-Qura University, Makkah, Saudi- \\ Arabia \\ ${ }^{3}$ Department of Chemistry, Faculty of Science, Mansoura University, El-Gomhoria Street, Egypt \\ *E-mail: n_elmetwaly00@yahoo.com; nmmohamed@uqu.edu.sa
}

doi: $10.20964 / 2021.06 .30$

Received: 11 January 2021 / Accepted: 30 March 2021 / Published: 30 April 2021

Cyclopentolate (CLO) is an antimuscarinic compound with actions similar to atropine which induces both mydriasis and paralysis of the ciliary muscle. The present work describes the fabrication of novel carbon paste electrodes (CPE) incorporated with zeolite as a promising analytical protocol for sensitive voltammetric assay of cyclopentolate. Remarkable enhancement of the sensitivity towards CLO was achieved compared with the blank electrode. An irreversible oxidation process with the two-electron transfer was reported elucidated with molecular orbital calculations. The recorded peak currents were linearly proportional to the CLO within the concentration range 48.03 to $725.00 \mathrm{ng} \mathrm{mL}^{-1}$ with LOD of $14.53 \mathrm{ng} \mathrm{mL}^{-1}$. High sensitivity, fabrication reproducibility, and stability were achieved. Noticeable resolution between the cyclopentolate and phenylephrine voltammetric peaks offers simultaneous assay of cyclopentolate in different pharmaceutical formulations in presence of phenylephrine, excipients, or CLO degradation products. The achieved sensitivity and selectivity demonstrate the applicability of zeolite based sensor for determination of cyclopentolate with average recoveries agreeable with the official pharmacopeial methods.

Keywords: Cyclopentolate hydrochloride; Differential pulse voltammetry; Carbon paste electrode; Zeolite Y; Molecular orbital calculations.

\section{$\underline{\text { FULL TEXT }}$}

(C) 2021 The Authors. Published by ESG (www.electrochemsci.org). This article is an open access article distributed under the terms and conditions of the Creative Commons Attribution license (http://creativecommons.org/licenses/by/4.0/). 